been reported to range widely from 11-78\%. Full publication is important and for the aspiring trainee enhances there CV. However it may be difficult to publish a negative study, with service review papers also suggested to be less likely to achieve full publication. This study evaluates the rate of BSG abstracts subsequently published in full over a 15-year period and the time duration between meeting and full publication.

Methods All abstracts presented at the BSG between 1994 and 2008 were assessed in Nov 2012. This ensured a 4 year period had elapsed since the last meeting included this study, a previously reported upper limit timescale of full publication of an abstract after a meeting. PUBMED and EMBASE databases were reviewed using cross-referencing of first author, senior author and at least one key word from the abstract title. Abstracts and possible full publications were then examined in tandem to ensure they represented the same study. Full publication rates and lag time were then compared between meetings, with an unpaired test used to compare means and categorical data compared using a $\mathrm{X}^{2}$ test.

Results In order to provide comparable year on year data, outcomes of abstracts presented in the spring and autumn meetings of 1994 and 1995 were combined. Over the 15 -year period the number of abstracts presented ranged from $578-330$ but this did not vary significantly between years. However, the number ( $n=323-$ $\mathrm{n}=91)$ and percentage $(55.9 \%-20.4 \%)$ of abstracts presented that went on to full publication fell year on year $(r=-0.74 ; p=0.002$ and $r=-0.83 ; p=<0.001$ respectively). Comparing lag times between meeting and full publication at the start of the study period in 1994 (mean 23.0 months: SD 15.04) and at the end in 2008 (mean 19.6 months: SD 9.2), this was significantly longer $(p=0.014$ unpaired $t$ test). Service development abstracts had a conversion rate to full publication of $6.9 \%(8 / 116)$ between $2004-$ 2008, which was significantly lower than the $23.1 \%$ (525/2268) conversion rate identified for all abstracts submitted to the BSG during the same period $(p<0.0001)$

Conclusion This study demonstrates that the number of abstracts that go on to achieve full publication at the BSG has fallen with a significant trend. Whilst improvements are identified in the time to full publication, the decline in BSG abstract output to full publication may reflect declining research activity within the UK gastroenterology community.

Disclosure of Interest None Declared

\section{PTU-010 PERFORATION OR MUCOSAL TEAR- A CLASSICAL PRESENTATION OF COLLAGENOUS COLITIS WITH MUCOSAL TEAR}

doi:10.1136/gutjnl-2013-304907.103

1,"M Rajaram, ${ }^{2} \mathrm{~S}$ Ramasamy, ${ }^{2} \mathrm{~K}$ Koss. 'Gastroenterology, Nottingham University Hospital NHS Trust, Nottingham; 'astroenterology, East Cheshire Hospital NHS Trust, Macclesfield, UK

Introduction In general, the colonic mucosa is macroscopically normal in collagenous colitis, although minor, non-specific abnormalities may be found. Significant endoscopic abnormalities, «mucosal tears» representing longitudinal mucosal lacerations, have been reported in a few patients with collagenous colitis (1). The colonoscopist should aware that the risk of perforation is likely to be increased when mucosal tears are present.A 56-year-old female patient was referred to the outpatient clinic with 6 weeks history of profuse watery diarrhoea. Her significant past medical history includes colonic polyps and polypectomy in 2000 and her last colonoscopy in 2003 was normal. Her drug history includes alendronic acid $70 \mathrm{mg}$ once a week, hormone replacement therapy patches, solifenacin $5 \mathrm{mg}$ once a day and loperamide $2 \mathrm{mg}$ as required. Her initial blood investigations including FBC, Urea \& electrolytes, LFT, TFT, inflammatory markers, coeliac serology
\& stool cultures were normal. Her colonoscopy revealed a mucosal tear at the splenic flexure and the biopsies confirmed collagenous colitis.

Methods N/A
Results N/A

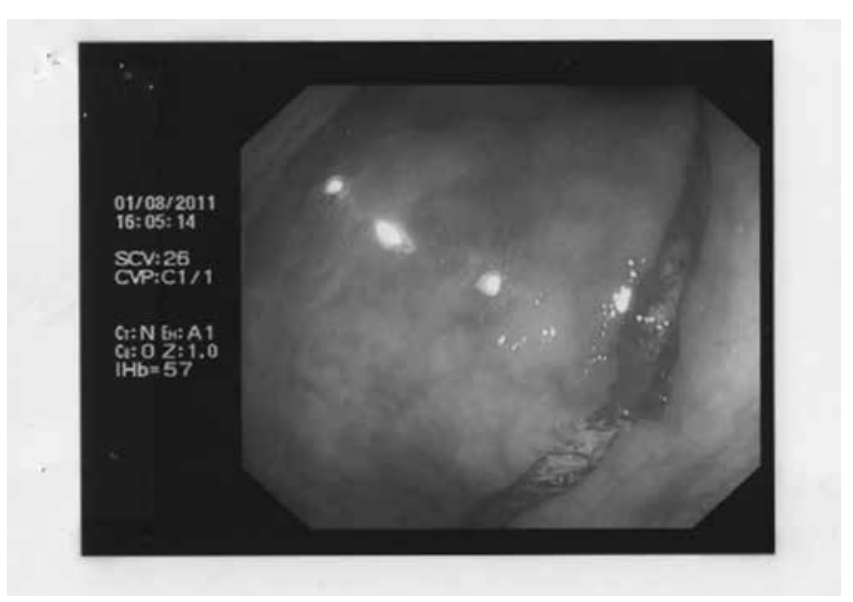

\section{Abstract PTU-010 Figure}

Conclusion The colonoscopy usually reveals a normal mucosa or mild mucosal edoema \& usually biopsies required to confirm the diagnosis. The mucosal tears on endoscopic insufflation are not uncommon with collagenous colitis.A postulated mechanism for the mucosal lacerations in collagenous colitis might be the rigid character of the collagen containing subepithelium, possibly in combination with endoscopic insufflation,leading to stretching of the mucosa and subsequent tearing (2)

Disclosure of Interest None Declared

\section{REFERENCES}

1. Colonic mucosal tears in collagenous colitis. Wickbom A, Lindqvist M, Bohr J, Ung KA, Bergman J, Eriksson S, Tysk C.SourceDivision of Gastroenterology, Department of Medicine, Orebro University Hospital, Orebro, Sweden.

2. Collagenous colitis with mucosal tears on endoscopic insufflation: a unique presentation. M Cruz-Correa, F Milligan, F M Giardiello, et al. Gut 200251: 600 doi:10.1136/ gut.51.4.600

\section{PTU-011 A SURVEY ON PROVISION OF TEACHING FOR NASOGASTRIC TUBE PLACEMENT AS PART OF THE CURRICULA OF UK MEDICAL SCHOOLS}

doi:10.1136/gutjnl-2013-304907.104

1.*P N Brennan, 'L L Wong, 'G Robins. 'Department of Gastroenterology, York Teaching Hospitals NHS Foundation Trust, York, UK

Introduction Nasogastric tube (NGT) insertion for enteral feeding is common practise, but is associated with the risk of tube misplacement or malposition in both the immediate and subsequent time points. ${ }^{1}$ Between 2005 to 2010, 21 deaths and 79 other cases of harm have been reported on the updated National Patient Safety Agency (NPSA) Alert (2011), "Reducing harm caused by nasogastric feeding tubes". In $45 \%$ of cases, misinterpretation of the chest X-ray was directly responsible for the harm inflicted.

The General Medical Council's (GMC) guidance for undergraduate education, "Tomorrow's Doctors (2009)" does not specify NGT placement as a core competency for a graduate or Foundation Trainee. $^{2}$

The purpose of this survey was to ascertain the number of medical schools in the United Kingdom (UK) which provide specific teaching on NGT placement and correct identification of tube position (using either $\mathrm{pH}$ method or clinical interpretation of a plain 
chest X-ray film). Furthermore, the authors sought to identify whether these medical schools included formative or summative assessment of the respective methods.

Methods All 30 GMC recognised Medical Schools within the UK were invited to participate by means of a standardised survey proforma. This proforma was emailed to relevant staff members who were either responsible for course development or were personnel within the clinical skills faculty.

Results To date, there has been a $57 \%(17 / 30)$ response rate (Table 1).

\section{Abstract PTU-011 Table 1}

\begin{tabular}{ll}
\hline & Number of Medical Schools \\
\hline Formal teaching on NG tube insertion provided & 10 \\
Methods employed in assessing appropriate tube position: & \\
pH Testing & 10 \\
CXR interpretation & 6 \\
\hline
\end{tabular}

Of the 10 medical schools that provide formal teaching on NGT insertion, 8 of them required formative assessment for both the practical technique and the interpretation of correct tube position.

Of the 7 medical schools that do not to provide formal teaching of NGT insertion, 6 of them stated that this was due to the GMC's "Tomorrow's Doctors" guidance not including NGT placement as a mandatory proficiency for a graduate.

Conclusion This survey found that 59\% (10/17) of the participating UK medical schools provide formal teaching on NGT placement and correct identification of tube position. There is variable emphasis on NGT procedure proficiency in undergraduate medical education. Given the risks highlighted by the 2011 NPSA report, we would suggest that NGT placement instruction and training should be facilitated at the undergraduate level.

Disclosure of Interest None Declared

\section{REFERENCES}

1. National Patient Safety Alert (PSA 002): Reducing the harm caused by misplaced nasogastric feeding tubes in adults, children and infants; Mar 2011. URL: http:// www.nrls.npsa.nhs.uk/alerts/?entryid45 $=129640$

2. General Medical Council (GMC) Tomorrow's Doctors 2009; URL: http://www .gmc-uk.org/TomorrowsDoctors_2009.pdf_39260971.pdf

\section{PTU-012 ENDOSCOPY TRAINING USING SIMULATORS: WHAT FACTORS AFFECT TRAINEE MOTIVATION TO LEARN?}

doi:10.1136/gutjnl-2013-304907.105

1."R E Swann, ${ }^{2}$ A Renwick. 'Gastroenterology, NHS Greater Glasgow and Clyde, Glasgow; ${ }^{2}$ General Surgery, Royal Alexandra Hospital, Paisley, UK

Introduction Simulation is increasingly being advocated to enhance training in complex technical skills such as endoscopy. While a growing body of evidence suggests simulation use within a study context can improve technical and non-technical skills [1], little attention has been paid to the learning experiences of trainee doctors using simulators to learn such skills.

Motivation is an important component of effective learning but anecdotal evidence suggests some trainees may be less motivated to learn in a simulated environment than in traditional training.

Aim To determine which factors trainee doctors identified as positively and negatively affecting levels of motivation during endoscopy training using a simulator.

Methods We invited a cohort of surgical and medical gastroenterology trainees to participate in semi-structured interviews. 6 trainees who had used an endoscopy simulator as part of their regular training were purposively selected to include a range of stages of training. We analysed the recorded interviews in an inductive fashion from an interpretivist perspective concentrating on key themes, outlying cases and use of language to develop insight into factors affecting motivation.

Results Major emergent themes specific to simulator use included:

- Context of simulator use including: Physical environment, professional and educational

- Positive impact of intermittent rather than continuous supervisor presence and feedback

- Recognising limitations of utility

Differences in individual preferences relating to the above issues was also emphasised suggesting, where possible, a tailored approach would maximise motivation. The limitations of simulator based learning was repeatedly mentioned by senior trainees, with a suggestion that being forced to learn on simulators beyond a point where they were perceived useful could have a detrimental impact on motivation. However, several trainees identified alternative uses such as team training exercises which could provide motivating simulator based learning at more senior levels.

Conclusion The above issues should be considered in all units using simulators in training for endoscopy or similar complex technical skills. The impact of the physical environment, altered role of the supervisor and optimum methods of delivering feedback in simulator based endoscopy training merit further exploration.

As simulator based learning becomes more widely available, careful consideration of how it is used at different stages of the endoscopy curriculum is required.

Disclosure of Interest None Declared

\section{REFERENCE}

1. Cochrane Database Syst Rev. 2012 Jun 13; 6:CD008237

\section{PTU-013 THE IMPACT OF EDUCATION ON FLUID PRESCRIBING AMONGST JUNIOR DOCTORS}

doi:10.1136/gutjnl-2013-304907.106

1,*S Biswas, ${ }^{2} \mathrm{M}$ Stroud, ${ }^{1}$ A de Silva. 'Gastroenterology, Royal Berkshire Hospital NHS

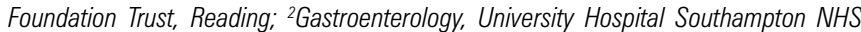
Foundation Trust, Southampton, UK

Introduction Gastroenterology patients frequently require intravenous fluids but inappropriate prescription is associated with poorer outcomes (1). Educational initiatives can improve fluid prescription (2) and this study examines knowledge of electrolyte requirements and post-operative fluid prescription amongst final year medical students and junior doctors, ten years on from the paper by Lobo et al (3).

Methods 56 junior doctors and final year medical students in a large UK teaching hospital answered a questionnaire on fluid prescribing practise before attending a formal teaching session on the topic where they also received a pocket handbook on Trust prescribing guidelines. Six months later they were asked to repeat the questionnaire. There were 36 respondents after the teaching session.

Results Prior to the teaching $25 \%$ did not feel confident with fluid prescribing and $34 \%$ felt they had unsatisfactory or poor teaching at medical school. $18 \%$ stated that fluid balance charts were not checked regularly. Only $10 \%$ of respondents were aware of the sodium content of normal saline and $9 \%$ would prescribe two or more litres of normal saline per day in a post-operative patient.

After the teaching session $94 \%$ felt confident with fluid prescribing and $64 \%$ knew the sodium content of normal saline. $5 \%$ would prescribe two or more litres of normal saline per day in a postoperative patient.

Conclusion Knowledge of electrolyte requirements and fluid prescribing is still inadequate, but a teaching session with a handout significantly improved knowledge and confidence in prescribing. The six months of experience gained in this time may be a confounding factor, but as the questionnaires indicated lack of fluid 